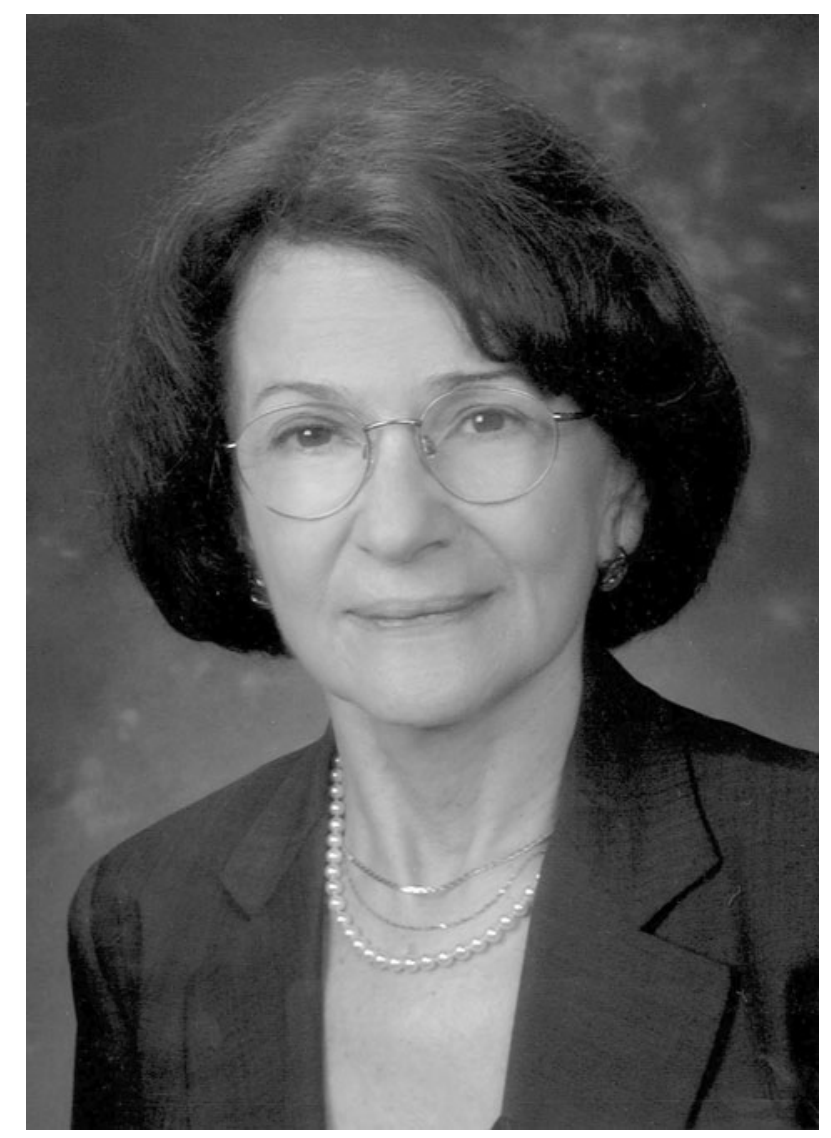

\title{
In Memory of \\ Professor Patricia S. Goldman-Rakic
}

In October of 1990, I and my colleagues Ed Smith and Bob Koeppe attended an ONR-sponsored conference on biological mechanisms of cognitive processes. At that conference, I was introduced to the work of Patricia Goldman-Rakic, who presented a riveting review of the work that she and her colleagues had conducted on working memory. Pat's use of single-cell and lesion techniques that focused on regions around the principal sulcus in monkeys had revealed remarkable computational prowess of single neurons in the service of storage in working memory. Readers of this journal do not need to be told the substance of this story: They will know that Pat's work (as well, of course, as the work of other pioneers in this area, such as Joaquin Fuster), extending, refining, and elaborating on other work beginning with that of Jacobsen, had unveiled remarkable delay-related activity in these neurons that provided crucial information about their role in preserving representations of space for short periods of time. My colleagues and I responded to this remarkable line of work by huddling in the hallway during conference breaks and concocting an experiment that might bring Pat's work to the human, via neuroimaging methods (at the time, PET). Our own research then jumped off from this point to issues of storage and manipulation of information in working memory, lines of research that have been pursued by many other investigators since. 
What we know about working memory from both the human and animal studies from the ensuing 14 years is a vast expansion upon what we knew in 1990. The psychology behind Pat's work had offered many clues about processing mechanisms underlying working memory, but it is in large part the research of Pat Goldman-Rakic, her predecessors, her collaborators, and her contemporaries that stimulated the vast body of research that has mapped out the brain mechanisms that we know about today. To be sure, the past 14 years of research have raised as many questions as they have answered, about the nature of ever increasingly detailed knowledge about a psychological system. Indeed, the papers included in this issue reflect the broad reach of Pat's work and her influence, and they give a sense of just how much impact her contribution has had.

It has been over a year since psychology and neuroscience lost the promise of future contributions that Pat Goldman-Rakic would make. We thought this an appropriate opportunity to present a palette of research findings that have been stimulated by her seminal research, to present work by some of her collaborators and students, and to generally commemorate the huge contributions of this important figure in psychology and neuroscience. Our inclusion of papers here is not meant to be representative or encyclopedic. That would be simply impossible in a publication of such limited length. Rather, we hope to induce a moment of reflection about the contributions and influence of Professor Goldman-Rakic and perhaps to stimulate further interest in the sorts of problems that stimulated me and my colleagues. We also hope to highlight how important her multidisciplinary approach is in the study of difficult problems of memory, the frontal lobes, and psychopathology that engaged her.

We have the deepest respect for the lasting legacy that Patricia Goldman-Rakic left us.

John Jonides

Ann Arbor, Michigan

December 1, 2004 